\title{
Study on the mixing performance of one differential twin-screw kneader
}

\author{
Jing WEI *, Dabing CHEN** and Dongming ZHOU** \\ *The State key Laboratory on Mechanical Transmission Chongqing University \\ Chongqing Shapingba District sand 174 Center Street, Chongqing City 400030, China \\ weijing_slmt@163.com \\ **School of Mechanical Engineering Dalian University of Technology \\ No.2 Linggong Road, Ganjingzi District, Dalian City, Liaoning Province, PRC, 116024, China
}

Received 24 September 2014

\begin{abstract}
Twin-screw kneader is an efficient polymer processing equipment. The mixing performance is a very important index for the twin-screw kneader. In this paper, the mixing performance of one novel intermeshing counter-rotating twin-screw kneader with different tip angles of the male rotor was simulated using the mesh superimposition technique (MST). Statistical analysis was carried out for the flow field using particle tracking technique, and distributive mixing performance is evaluated using the residence time distribution and segregation scale, while the dispersive mixing performance is estimated using the average of the maximum shear rate and stretching rate as well as the mixing index. The results show that the best distributive mixing performance is achieved when the tip angle is $0^{\circ}$, while the optimal dispersive mixing performance is obtained when the tip angle is $20^{\circ}$. One experimental apparatus of the twin-screw kneader is built up to perform the mixing performance using three color masterbatch materials with the screw rotors. The results in this paper provide a data basis for the selection of parameters and optimization of the mixing performance for the differential twin-screw kneader.
\end{abstract}

Key words: Tip angle, Twin-screw kneader, Rotor profiles, Distributive mixing performance, Dispersive mixing performance

\section{Introduction}

Kneaders are closed operation units based on a self-cleaning mechanism (Böhme. and Wünsch, 1997). Nowadays, the kneaders are widely used in blister forming and extrusion forming as well as in chemical industry, rubber industry or pharmaceutical industry for various purposes. As a continuous high efficient polymer processing mixer, especially with the rapid development of modern high polymer synthesis industries, kneaders are widely used as the core equipment of polymer reactors (Huang, et al.,2006; Shon, et al., 2008).

Recently, twin-screw kneaders, as one type of kneaders, are used more and more in polymer processing. The main functions of the twin-screw kneader are self-cleaning, conveying, mixing and melting, shearing, rolling. The core component of twin-screw kneader is one pair of intermeshing screw elements. As is known to all, for the conventional screw elements of co-rotating or counter-rotating twin-screw kneaders, they have identical end cross-sections and rotate at the same speed, when the teeth number is odd, the two screw elements have the same position layout, while there is a phase difference between the screw elements when the teeth number is even. With the rapid development of the polymer industry, higher request is put forward to the twin screw kneader, such as higher melting efficiency, better mixing quality etc., while the conventional screw elements are increasingly unable to meet these requirements. Therefore, screw elements have been emphasized on and developed continuously, and different types of screw elements have been invented in recent years, such as the screw mixing element (SME), a kind of special element, was a distributive mixing promoter consisting of a standard screw profile with slots cut across the flight tip to increase leakage flow (Ishikawa, et al., 2006), the MR, ML and MPE mixing elements manufactured by Berstorff (Potente, et al., 2001) and combing mixer elements(GFM) (Djuric and Kleinebudde, 2008), ZME/TME components (Brouwer, et al., 2002) 
etc., which effectively improve the mixing quality and efficiency of the twin-screw kneader and greatly promote the development of polymer industry.

According to the current research, for traditional twin-shaft kneaders, the improvement of efficiency in intrinsic self-cleaning, transportation and mixture mechanisms is limited by the mechanical design itself. But these mechanisms can be improved by changing the profiles of twin-shaft kneaders based on the gear engagement theory. For screw pumps, the fill level of the plug-flow reactor depends on the intrinsic self-cleaning properties of the reactor itself. This phenomenon is well known as the "compulsory transportation effect" or "pumping effect". Based on these observations and working principles of traditional twin-screw kneaders, a novel continuous differential counter-rotating twin-screw kneader reactor, which is composed of self-cleaning, compulsory transportation and mixing and plasticizing, is proposed (Fig.1(a)), and the teeth number of the female and male rotors is four and one respectively (Wei, et al., 2010). The working principles of the twin-screw kneader are analyzed and numerical simulations for dynamic characteristics of the flow field are carried out. Three-dimensional, isothermal and steady state numerical analysis of a non-Newtonian fluid is presented based on computational fluid dynamics (CFD) theory, and the characteristics under the conditions of different speeds and center distances are studied (Wei, et al., 2014). The static loads on twin-screw kneaders were studied and a mathematical model of the force and torque moments using a numerical integration method based on differential geometry theory is presented (Wei, et al., 2008).

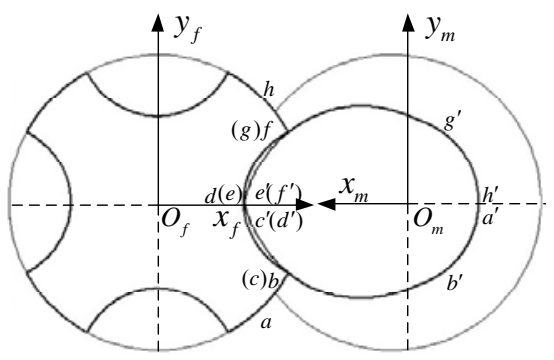

(a)

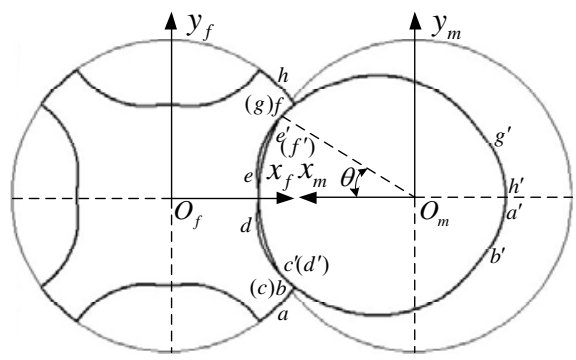

(b)

Fig. 1 End cross-section of screw rotors: (a) End cross-section of screw rotors of a novel twin-screw kneader, (b) original end cross-section of screw rotors

In actual application process, two or more polymers are usually mixed to achieve the performance requirements of the final product in order to eliminate the performance deficiencies since single polymer cannot meet with the materials performance requirements. The morphology and the specific properties of the final product depends on the uniformity of the mixture and therefore on the quality of the mixing. Mixing is comprised of distributive mixing and dispersive mixing, and distributive mixing, achieved by frequent rearrangement and reorientation, is used to describe the distribution of different components in the volume under consideration, while dispersive mixing, obtained by the shear and elongational effect, involves disaggregating or dispersing solid particles, liquid droplets, or gas bubbles . Both goals can be achieved simultaneously or in several steps in the mixer.

Nowadays, two methods are widely used to study the mixing performance. The first is experimental approach, while the other is the numerical method. Ishikawa, et al.,(2000) adopted the experimental method and the numerical method respectively to analyze the mixing performance of mixer. Due to the complexity of the flow field and the limitations of experiment, it is almost impossible to obtain all local information by using the experimental approaches, and experimental approaches are usually very expensive and time consuming. It is an effective way to study the mixing performance by using professional fluid simulation software, such as FIDAP, POLYFLOW, FLUENT. Numerical simulation methods not only have the advantages of low cost and fast speed, but also can solve those problems that are difficult to solve by experiments.

In the process of researching the mixing performance, influence of the proportion of different materials, screw speed, center distance, screw pitch and other design and process parameters on the mixing performance is usually studied by the researchers. T. Ishikawa et al have evaluated the tip-clearance effect on mixing at the kneading block section of one co-rotating twin-screw extruders using 3-D numerical simulations based on finite element technique with the marker particle tracking analysis (Ishikawa, et al., 2006). Emin and Schuchmann (2013) have simulated the flow of plasticized maize starch in a co-rotating twin-screw extruder using computational fluid dynamic (CFD), and the flow type and profile in the extruder were discussed by using the influence of screw speed and screw configuration as exemplary process parameters. In order to determine the flow patterns in the extruder, the governing equations were 
calculated by CFD software POLYFLOW, and mesh superimposition technique (Avalosse, et al., 2002) was introduced to avoid the use of a remeshing algorithm. The mesh superimposition technique involves separately meshed flow domain, moving elements and superimposes them at time intervals with a penalty formulation of the governing equations, and the method is robust since no remeshing algorithms are needed. Alsteens, et al., (2004) analyzed the effect of the material and the geometrical parameters of the kneading block, such as the stagger angle and the width of the discs, on the mixing efficiency of twin-screw extruder with three-dimensional time-dependent finite element calculations implemented by POLYFLOW, and the robustness and the accuracy of the mesh superimposition technique (MST) is evaluated. simulated and analyzed the mixing performance of the novel twin screw kneader (illustrated in Fig. 1(a)) using finite element method (FEM) combined with MST, and discussed the influence of center distance, lead and speed of rotor on the mixing performance (J.Wei, et al., 2014). But for the design parameter of the tip angle, which directly determines the size of the screw edge region when the tip circle radius of the screw is given, not much published work is available on the analysis of its influence on the mixing performance.

In this paper, the object of this work is to evaluate the sensitivity of tip angle on the mixing performance of screw rotors for above proposed novel twin-screw kneader using FEM along with MST as implemented by POLYFLOW to calculate the residence time distribution (RTD), segregation scale, shear rates, stretching rates and mixing indexes during the mixing process.

\section{Numerical models and boundary conditions \\ 2.1 Geometry Model}

When the profiles at the end cross-section of female and male rotors rotate along their center axes with a certain lead, a three-dimensional solid model of the screw rotors can be obtained. The parametric equations of a right-directional helicoid can be expressed as:

$$
\left\{\begin{array}{l}
x=x(t) \cos \tau-y(t) \sin \tau \\
y=x(t) \sin \tau+y(t) \cos \tau \\
z=P \tau \\
P=S / 2 \pi
\end{array}\right.
$$

where, $x(t)$ and $y(t)$ are the coordinates of the profiles at the end cross-section of female and male rotors. $\tau$ is a parametric variable, which shows the rotation angle from the initial position to the end position along axis $Z, S$ is the lead of the screw rotors, for male and female rotor, $S$ is $S_{M}$ and $S_{F}$ respectively. Here we take the clockwise direction as positive value, for left-directional helicoid, the positive sign in front of $\tau$ in the equations of $x$ and $y$ needs to be changed to negative sign. According to the equations of helicoid and the coordinates of the profiles at the end cross-section of the rotors, the 3D model of the female and male rotors can be obtained.

According to Fig.1, the coordinates of the profiles at the end cross-section of female and male rotors are listed in authors' previous published papers (2010 in J. Reinforced Plastics and Composites, 2014 in Polymer Engineering and Science). Fig. 2 shows the geometry models of female and male rotors when the tip angle is $0^{\circ}, 20^{\circ}$ and $40^{\circ}$ respectively. As can be seen, with the increasing of tip angle $\theta$, dimension of the screw groove increases and the size of the screw edge region decreases gradually of the female rotor, while the male rotor is opposite. So it is clear that tip angle of the male rotor directly affects the dimensions of screw groove and screw edge region, which are the important parameters in the design of screw rotors. Therefore, in order to design screw rotors with reasonable structure and ideal performance, study of the relationship between the tip angle and the mixing performance will be helpful.

In this paper, we select five groups of tip angles, which are $0^{\circ}, 10^{\circ}, 20^{\circ}, 30^{\circ}$ and $40^{\circ}$ respectively, to implement the comparative analysis of the mixing performance of screw rotors. 


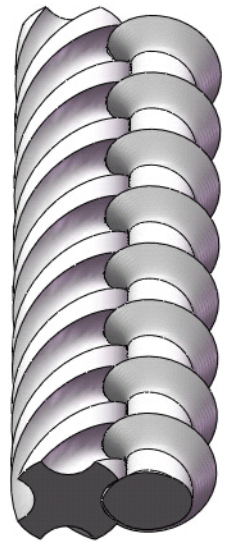

(a)

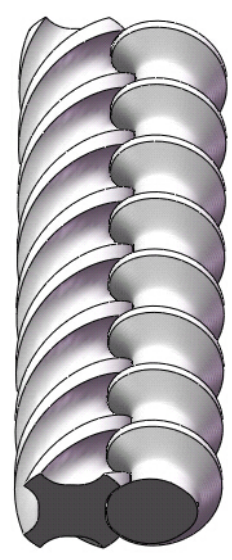

(b)

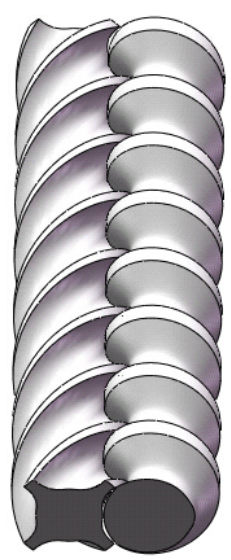

(c)

Fig 2 Geometry models of the screw rotors: (a) $\theta=0^{\circ}$, (b) $\theta=20^{\circ}$, (c) $\theta=40^{\circ}$.

\subsection{Finite Element Model}

POLYFLOW is a finite element computational fluid dynamics (CFD) program designed primarily for simulating applications where viscous and viscoelastic flows play an important role. The flows can be isothermal or non-isothermal, two or three-dimensional, steady-state or time-dependent. POLYFLOW is usually used to solve flow problems in polymer and rubber processing, food rheology, glass-work furnaces, and many other rheological applications, and it has a wide variety of fluid models and enough constitutive models available.

After building the geometry models of screw rotors and flow channel, finite element mesh generation and specifying of the boundary and region types are done by GAMBIT, the preprocessor of POLYFLOW. In order to facilitate comparison, the identical method of mesh generation is applied in different tip angels. Since the simulation process used is the transient analysis, POLYFLOW will remesh the rotors and flow channel at each time step using MST. Fig.3 shows the finite element models of screw rotors and flow channel at $\theta=0^{\circ}$, the left female screw rotor and the right male rotor has 145,383 and 105,140 tetrahedral elements respectively, while there are a total of 128,000 hexahedron elements in the flow channel region with a three-layer boundary layer that has a thick equal to the clearance between the screw rotor and flow channel, and the intermeshing area has been refined in order to obtain a good result.

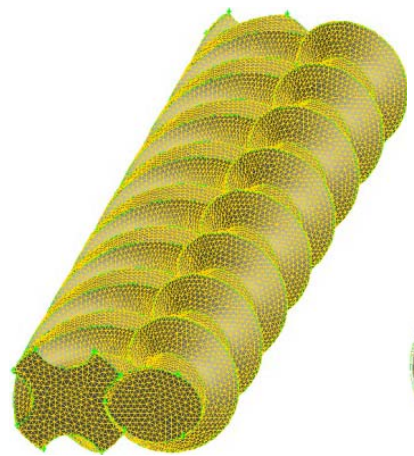

(a)

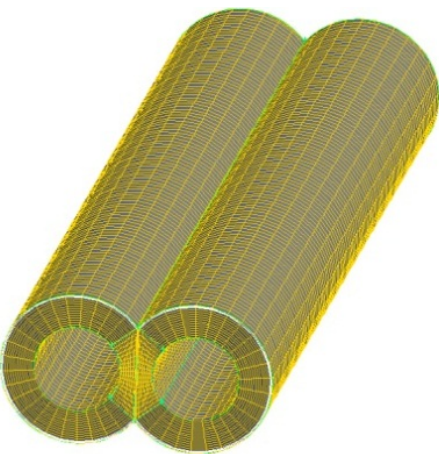

(b)

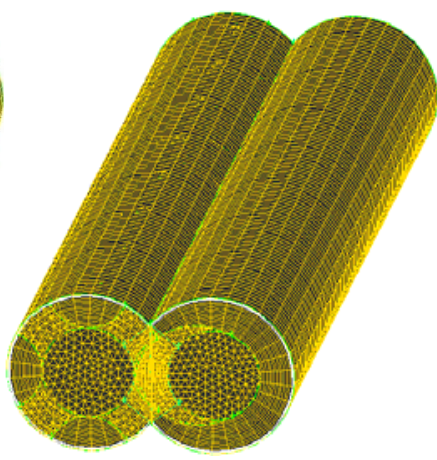

(c)

Fig. 3 Finite element models: (a) screw rotors, (b) flow channel, (c) superimposed screw rotors and channel.

\subsection{Mathematical Model and Boundary Conditions}

In the simulation process of flow field, taking the specific process conditions of melt conveying section and polymer properties into account, following assumptions are made (Kajiwara, et al., 1996)

(1) Material is fully filled in the entire flow channel.

(2) The fluid flow is isothermal, that is all the points have the same temperature in the flow field.

(3) The flow is laminar flow, and the Reynolds number is very small.

(4) Volume force, such as inertial force, gravity, is negligible, because it's much smaller than the viscous force.

(5) The fluid is incompressible. 
(6) There is no-slip on the wall of flow channel.

Based on the above assumptions, continuity equation, momentum equation and constitutive equation are given respectively to describe the fluid.

Continuity equation:

$$
\nabla \cdot v+\frac{\beta}{\eta} \Delta p=0
$$

Momentum equation:

$$
H(v-\bar{v})+(1-H)(-\nabla p+\nabla \cdot T+\rho g-\rho a)=0
$$

Constitutive equation:

$$
T=2 \eta D
$$

where, $\nabla$ is the Hamilton operator, $v$ is the velocity vector, $\beta$ is the relative compression factor, default is 0.01 in POLYFLOW, $\eta$ is the apparent viscosity, $p$ is the pressure, $H$ is a step function, value is 0 or $1, \bar{v}$ is the local velocity of moving parts, $T$ is the extra-stress tensor, $\rho$ is the density of material, $\rho g$ is the volume force, $\rho a$ is the acceleration term, $D$ is the rate of deformation tensor.

The fluid is non-Newtonian fluid and its constitutive equation follows Power-law model, which can be expressed as (Rathod and Kokini, 2013)

$$
\eta(\dot{\gamma})=K(\lambda \dot{\gamma})^{n-1}
$$

where, $K$ is the consistency factor,$\lambda$ is the natural time, and $n$ is the power-law index which is a property of the material.

In the simulation, physical parameters of polypropylene (PP), which is: $K=3802 \mathrm{~Pa} \cdot \mathrm{s}, \lambda=1 \mathrm{~s}, n=0.38$, are used.

During the simulation, the female rotor rotates counter-clockwise while the male rotor rotates clockwise, and the rotational speeds of them are $25 \mathrm{rpm}$ and $100 \mathrm{rpm}$ respectively. Since the rotation angle of male and female rotors are set as $9^{\circ}$ and $36^{\circ}$ respectively each time, so the time step is $0.06 \mathrm{~s}$. In the analysis process, we consider the screw rotors as the moving parts, due to no slip of the screw surface, then the melt speed of the screw surface is the same as that of the screw surface. As the barrel is a fixed component, so the speed of the outer wall of the flow channel which coincides with the barrel inner surface is zero. Inlet and outlet of the channel adopt the differential pressure boundary condition, that is $\Delta P=P_{0}-P_{\mathrm{I}}$, and the inlet pressure $P_{\mathrm{I}}$ and outlet pressure $P_{0}$ is $0 \mathrm{MPa}$ and $5.0 \mathrm{MPa}$, respectively.

\section{Results and discussions}

\subsection{The Spatial Flow Pattern of Particles in the Flow Channel}

For the dynamic simulation of mixing process, the lead of male rotor is $50 \mathrm{~mm}$, rotation speed is $100 \mathrm{rpm}$, since the ratio of teeth number of female and male rotors is four to one, the lead of female rotor is $200 \mathrm{~mm}$ and revolving speed is $25 \mathrm{rpm}$, center distance between the axes of female rotor and male rotor is $50.4 \mathrm{~mm}$. According to the simulation results, spatial flow patterns of particles in different tip angles are consistent, and here we give the spatial flow pattern when the tip angle is $0^{\circ}$ to describe this process.

At $t=0 \mathrm{~s}, 1500$ particles were evenly placed initially in the inlet of the flow channel, and these particles are considered to be massless points such that their presence does not affect the flow field and interactions among particles, such as Van der Waals attraction forces and friction are ignored (Cheng and Manas-Zloczower,1998). In order to facilitate the observation of particles mixing, a concentration field is created in the entry section, because there is no diffusion and chemical reactions between the particles, so the concentration of any particle is a constant, and the particles in the upper part of the inlet have a concentration 1 and their colors are red, while the particles in the lower part have a concentration 0 and their colors are blue. With the rotation of the screw rotors, tracer particles flow from inlet to outlet in the flow channel gradually. Fig. 4 records the movement situation of the particles from $\mathrm{t}=0 \mathrm{~s}$ to $\mathrm{t}=5.88 \mathrm{~s}$. At $\mathrm{t}=0.42 \mathrm{~s}$, it can be seen that the particles are pushed forward with the rotation of the screw rotors, meanwhile, there exist a mutual exchange of particles between the upper and lower part during the movement, but it is not obvious. At 
$\mathrm{t}=0.84 \mathrm{~s}$, we find that there are more blue particles moving into the upper part of the right side of the channel, but almost no particles move into the upper part of the left side of the channel, which is mainly because the rotational speed of male screw is significantly higher than that of the female screw, so the blue particles move into the upper part from the lower part of the channel in a shorter time under the driving of the male rotor. At $t=1.26 \mathrm{~s}$, some blue particles have moved into the upper part of the left side of the channel, which is rather obvious when $t=1.68 \mathrm{~s}$. As the time goes on, tracer particles continue to move in the flow channel and the particle exchange is more intensified. At $\mathrm{t}=4.62 \mathrm{~s}$, some tracer particles have reached the end of the flow channel and outflow from the outlet. At $t=5.88 \mathrm{~s}$, more tracer particles outflow from the outlet, and the two kinds of particles have reached a better mixing state as Fig.4(o) shows. The spatial flow pattern of particles in the flow channel indicates that particles not only exist the axial movement in the flow channel, but also there is cross flow between the upper and lower part. Therefore, the particles continuously exchange between the two sides of the flow channel, which can improve the distributive mixing capability.

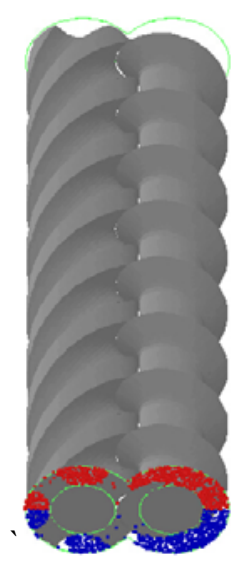

(a) $\mathrm{t}=0 \mathrm{~s}$

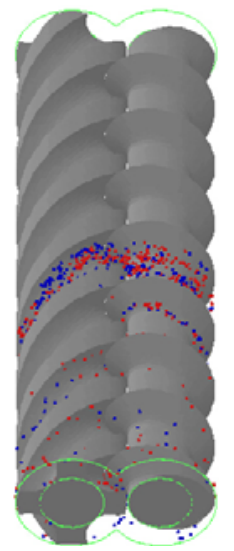

(f) $\mathrm{t}=2.10 \mathrm{~s}$

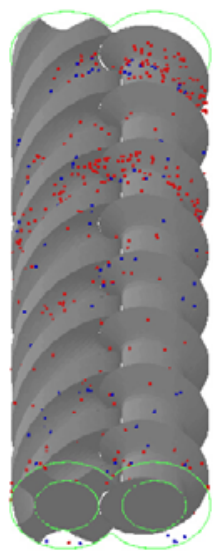

(k) $\mathrm{t}=4.20 \mathrm{~s}$

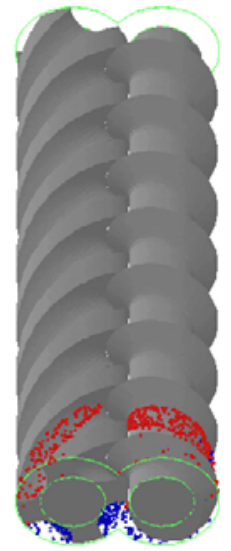

(b) $\mathrm{t}=0.42 \mathrm{~s}$

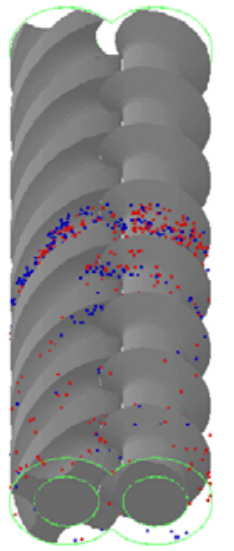

(g) $\mathrm{t}=2.52 \mathrm{~s}$

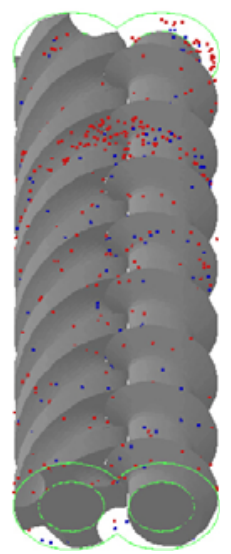

(1) $\mathrm{t}=4.62 \mathrm{~s}$

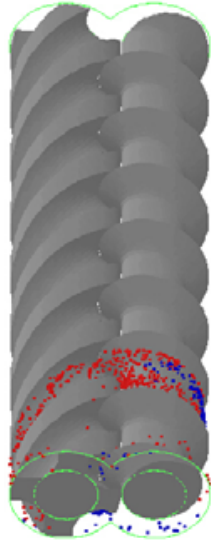

(c) $\mathrm{t}=0.84 \mathrm{~s}$

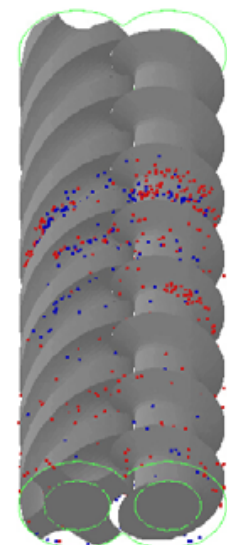

(h) $\mathrm{t}=2.94 \mathrm{~s}$

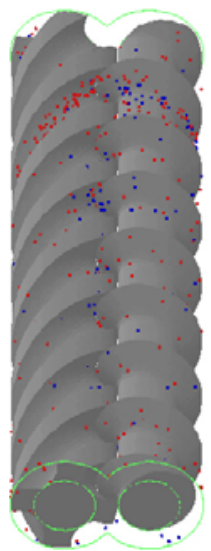

(m) $\mathrm{t}=5.04 \mathrm{~s}$

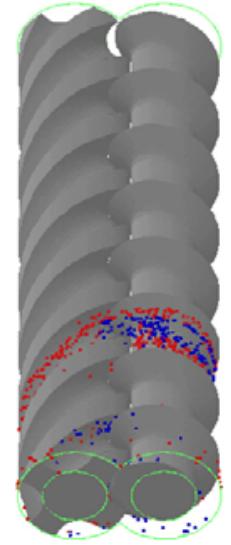

(d) $\mathrm{t}=1.26 \mathrm{~s}$

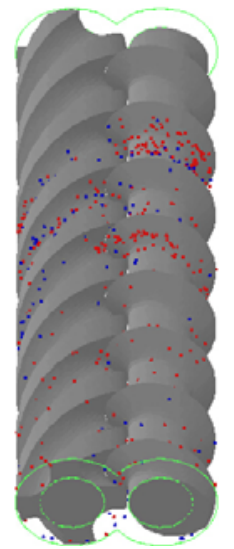

(i) $\mathrm{t}=3.3 \mathrm{~s}$

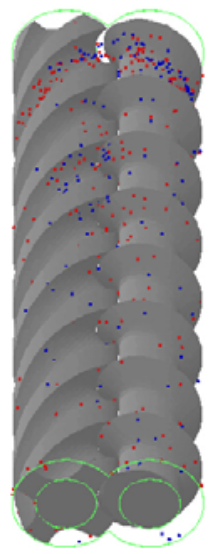

(n) $\mathrm{t}=5.46 \mathrm{~s}$

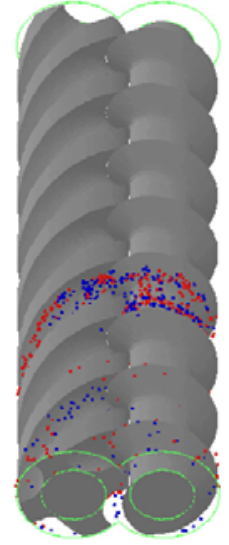

(e) $\mathrm{t}=1.68 \mathrm{~s}$

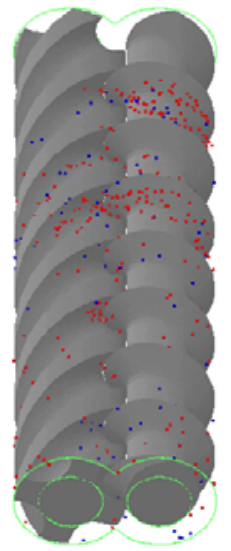

(j) $t=3.78 \mathrm{~s}$

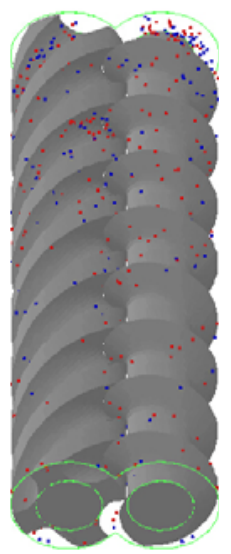

(o) $\mathrm{t}=5.88 \mathrm{~s}$

Fig. 4 The spatial flow pattern of particles 


\subsection{Evaluation of Distributive Mixing Performance with RTD}

Residence time distribution (RTD) is an important indicator to measure the distributive mixing performance (Kumar, et al., 2008). The residence time is usually used to describe the time history of a fluid inside a reactor. As the trajectories and the velocities along these trajectories are often different for the particles, so the residence time is not unique, and the longer the residence time is, the better the mixing effect is. Comparing the RTD curves under different tip angles can directly reflect the effect of distributive mixing under different conditions. The residence time distribution function and the cumulative residence time distribution function are given by Eqs. (21) and (22).

$$
\begin{aligned}
& E(t)=\frac{C}{\int_{0}^{\infty} C d t} \cong \frac{C}{\sum_{0}^{\infty} C \Delta t} \\
& F(t)=\int_{0}^{t} E(t) d t \cong \frac{\sum_{0}^{t} C \Delta t}{\sum_{0}^{\infty} C \Delta t}
\end{aligned}
$$

where, $C$ is the concentration of the tracer at the exit at time $t, \Delta t$ is the time step.

Fig.5 shows the RTD curves under different tip angles, as can be seen from the graph, the residence time of particles mainly concentrate in the convex region. When the tip angle is $40^{\circ}$, the convex part of the RTD curve is in the leftmost, followed by $30^{\circ}$, and the deviation extent of the bulges of RTD curve under other tip angles is relatively consistent. Moreover, the closer the bulge tends to the left, the shorter the residence time of most particles is, so we can preliminary judge that the distributive mixing capability is weak when the tip angle is $30^{\circ}$ and $40^{\circ}$.

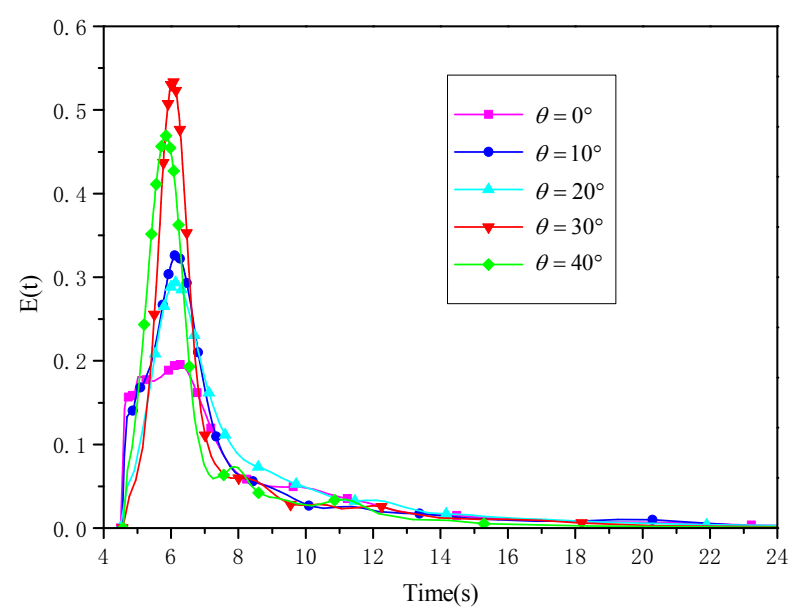

Fig. 5 RTD curves in different tip angles

In order to facilitate the quantitative analysis of residence time distribution, time of 90 percent of the particles outflow the channel under different tip angles, which is denoted by $T_{90}$, is counted. As shown in Fig. 6, it can be seen that $T_{90}$ is the longest when the tip angle is $0^{\circ}$, which indicates that the particles have experienced a quite long mixing process, and the distributive mixing ability is good, followed by $10^{\circ}$ and $20^{\circ}$, when the tip angle is $30^{\circ}$ and $40^{\circ}$, the corresponding value of $T_{90}$ is small especially when the tip angle is $40^{\circ}$, from which we can judge that the distributive mixing ability is weak when the tip angle is $30^{\circ}$ and $40^{\circ}$, and it is consistent with the above analysis results. 


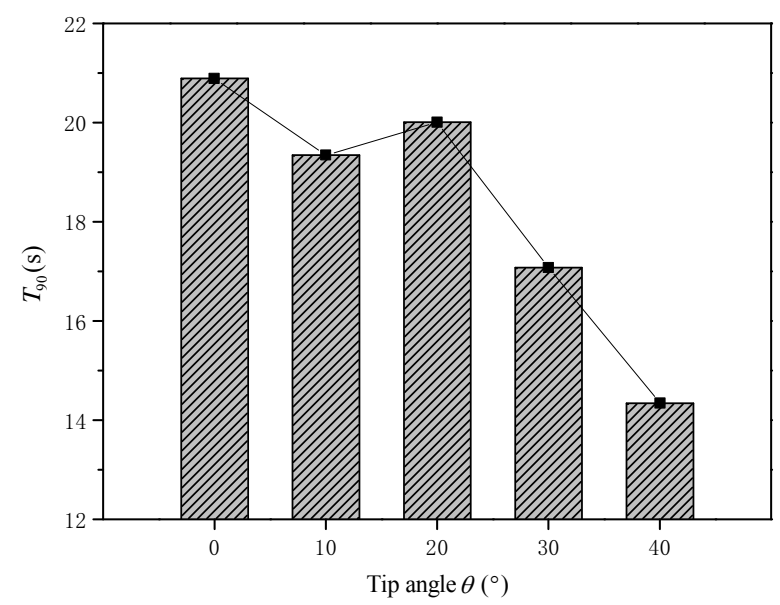

Fig. $6 T_{90}$ in different tip angles

\subsection{Evaluation of Distributive Mixing Performance with Segregation Scale}

Segregation scale is another important parameter in estimating the distributive mixing performance (Connelly and Kokini, 2007). In the process of describing the spatial flow pattern of particles, we defined the particles with a concentration 1 in the upper part of the channel inlet, while a concentration 0 in the lower part. Now, let $c$ denotes the concentration of particles in the mixing process, and its evolution is governed by $\dot{c}=0$.

At time $t$, consider a set of $M$ pairs of particles separated by a distance $r$. For the $\mathrm{j}$-th pair and time $t$, let $c_{j}^{\prime}$ and $c_{j}^{\prime \prime}$ denote the concentrations at both particles of the pair, moreover, let $\bar{c}$ denotes the average concentration of all particles and $\sigma_{c}$ the standard deviation. At time $t$, the correlation coefficient $R(r, t)$ for the concentration is defined as follows:

$$
R(r, t)=\frac{\sum_{j=1}^{M}\left(c_{j}^{\prime}-\bar{c}\right) \cdot\left(c_{j}^{\prime \prime}-\bar{c}\right)}{M \sigma_{c}^{2}}
$$

The function $R(r, t)$ gives the probability of finding a pair of random particles with a relative distance $r$ and with the same concentration. Let $\xi$ be such that $R(\xi, t)=0$, when $r=\xi$, we cannot predict whether the members of the pair have the same concentration or not.The segregation scale $S(t)$ is defined as shown in Eq.(24).

$$
S(t)=\int_{0}^{\xi} R(r, t) d r
$$

It is easy to understand that $S(t)$ is a measure of the size of the regions of homogeneous concentration, and $S(t)$ decreases when mixing improves.

Curves of segregation scale changing with time in different tip angles are shown in Fig.7. As can be seen, in the initial stage, the segregation scale gradually become smaller with time, after about $6 \mathrm{~s}$, the curves of segregation scale corresponding to $20^{\circ}, 30^{\circ}$ and $40^{\circ}$ appeare to fluctuate, and the values of segregation scale increases obviously, which indicate that particle accumulation phenomenon has emerged in the mixing process, and this phenomenon is not conducive to the distributive mixing of particles. When the tip angle is $0^{\circ}$ and $10^{\circ}$, the segregation scale curves also have the rising trend, but finally it tend to be stable, and the value is smaller.

Based on above analysis, the comprehensive judgment is obtained: when the tip angle is smaller than $20^{\circ}$, the distributive mixing ability of screw rotors is better than that while the tip angle is greater than $20^{\circ}$, and the best distributive mixing ability of screw rotor can be achieved when the tip angle is $0^{\circ}$. 


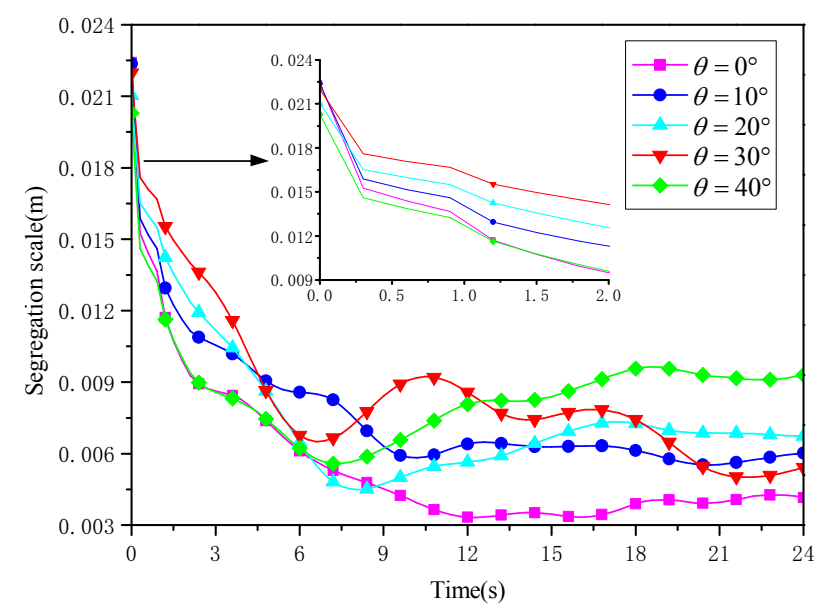

Fig. 7 Segregation scale in different tip angles

\subsection{Evaluation of Dispersive Mixing Performance with Shear Rate and Stretching Rate}

Sizes of material particles decrease continuously in the dispersive mixing process, and the shear rate and stretching rate plays a major role in the process, the larger shear rate and stretching rate can effectively promote the dispersion of particles. Fig. 8 shows the probability density function curves of maximum shear rate and stretching rate respectively, and it can be seen that this two sets of curves have the similar distribution trend respectively, but it's important to note the difference of peak in each set of curves, for example, when the tip angle is $20^{\circ}$, its corresponding peak is the smallest in each set of curves, which means at this time the number of particles with low maximum shear rate and stretching rate is the least, so there will be many particles subject to a high maximum shear rate and stretching rate, and the dispersive mixing performance will be better.

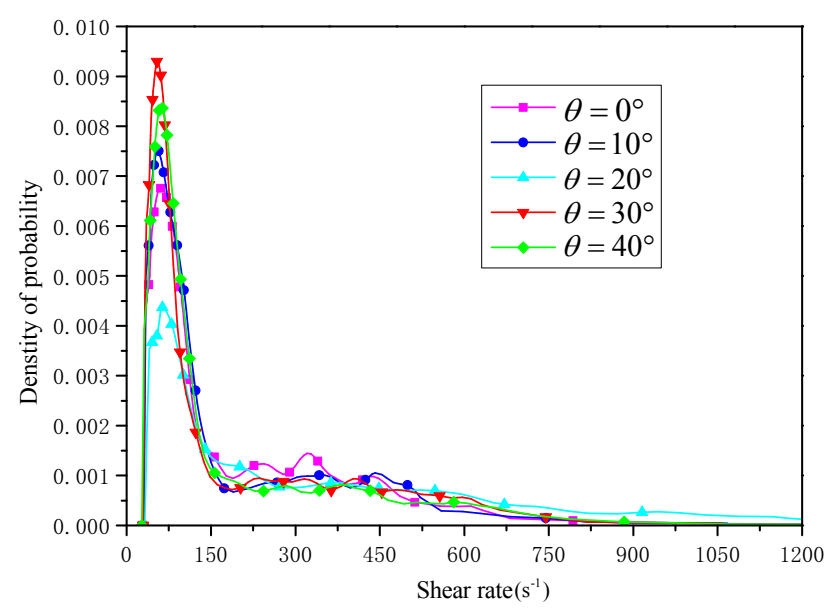

(a) 


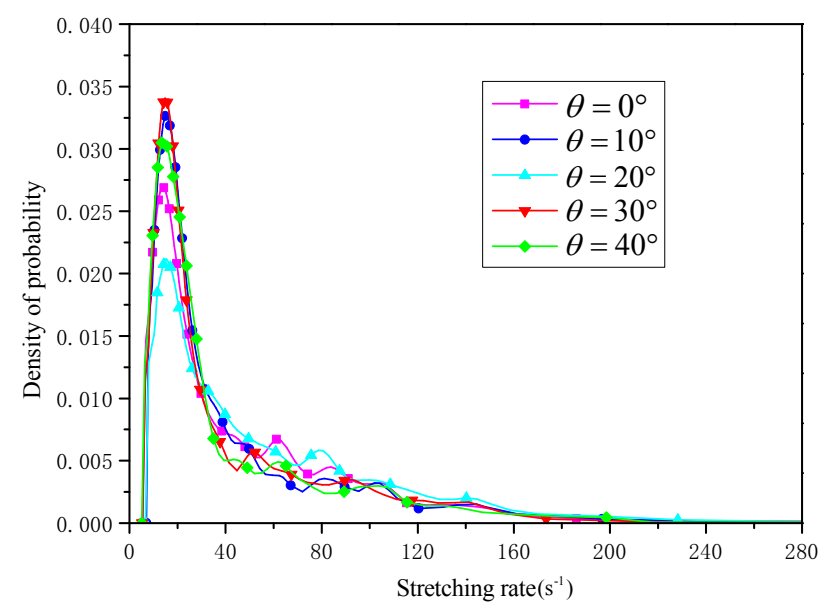

(b)

Fig. 8 Probability density function in different tip angles: (a) maximum shear rate, (b) maximum stretching rate.

In order to study the overall dispersive mixing performance of screw rotors in different tip angles, the average of maximum shear rate and stretching rate is solved. As shown in Fig. 9, it can be seen that when the tip angle is $20^{\circ}$, its corresponding value is obviously higher than that in other angles, which indicates that the particles have subjected to a stronger shear and stretching effect at this time, and the dispersive mixing performance of the screw rotor will be better. Moreover, when the tip angle is less than $20^{\circ}$, the corresponding value is higher than that when the tip angle is greater than $20^{\circ}$, which indicates that when the tip angle is less than $20^{\circ}$, the particles have experienced higher shear and stretching effect in the mixing process, and the difference of dispersive mixing performance in different tip angles will be further discussed below.

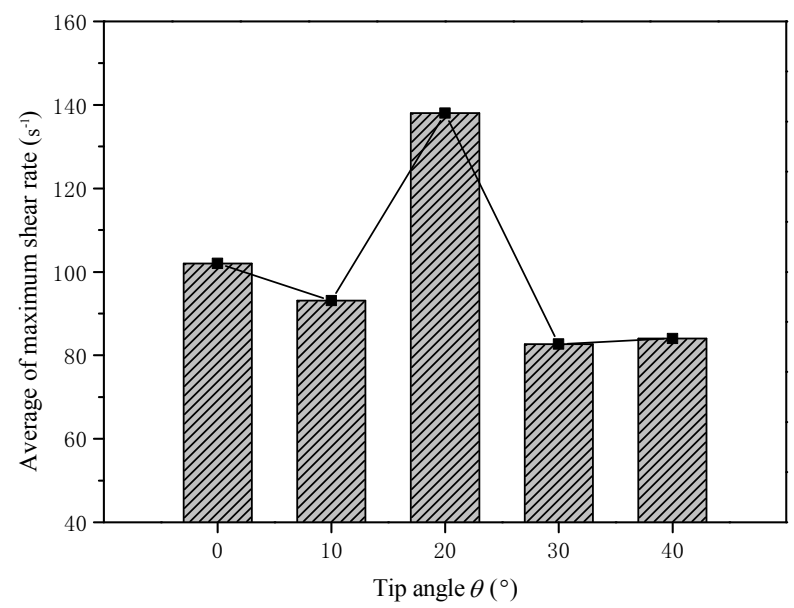

(a)

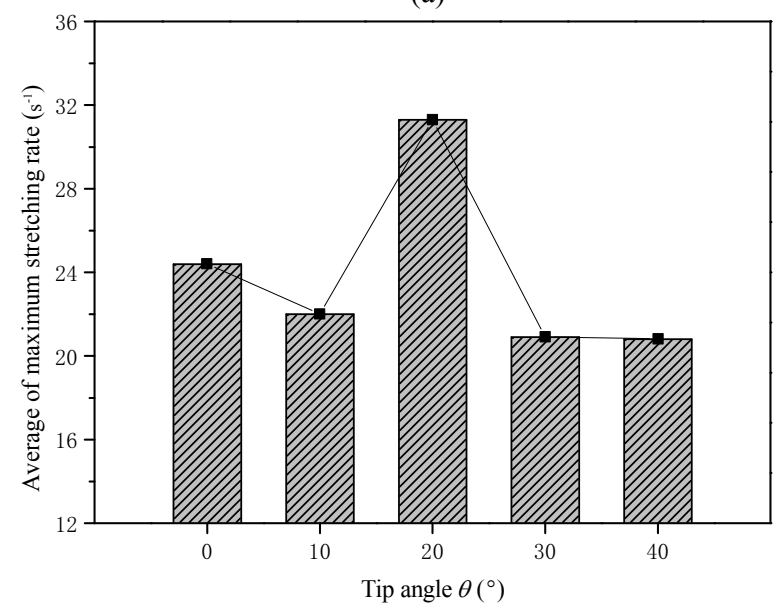

(b)

Fig. 9 Average in different tip angles: (a) maximum shear rate, (b) maximum stretching rate. 


\subsection{Evaluations of Dispersive Mixing Performance with Mixing Index}

In order to further study the dispersive mixing performance of the screw rotors, the parameter of mixing index, which is introduced by Cheng and Manas-Zloczower (1990) and can be considered to be a mapping of the mechanism of dispersive mixing within the kneader, is used to evaluate the flow characteristics of the flow field. Its definition is shown in Eq.(25).

$$
\lambda=\frac{|D|}{|D|+|W|}
$$

Where, $D$ is the rate of strain tensor and $W$ is the vorticity tensor, which are the symmetric and asymmetric components of the velocity gradient tensor. For a mixing index of 0 , the system is undergoing purely rotational flow and no effective mixing can occur. A mixing index 0.5 denotes simple shear flow, while a value of 1.0 denotes elongational flow, and elongational flow is more effective than the simple shear flow in the process of dispersive mixing.

Similarly, the average of the maximum mixing index is calculated to estimate the overall dispersive mixing ability of screw rotor in different tip angles. As shown in Fig.10, we can see that when the tip angle is $20^{\circ}$, its corresponding value is the maximum, which indicates that the dispersive mixing performance is the best at this time, and it is consistent with the above analysis. Moreover, when the tip angle is less than $20^{\circ}$, its corresponding value is lower than that while the tip angle is greater than $20^{\circ}$, which is opposite to the change trend shown in Fig.10, and it shows that when the screw angle is less than $20^{\circ}$, the proportion of shear flow the particles experienced is higher than that while the tip angle is greater than $20^{\circ}$.

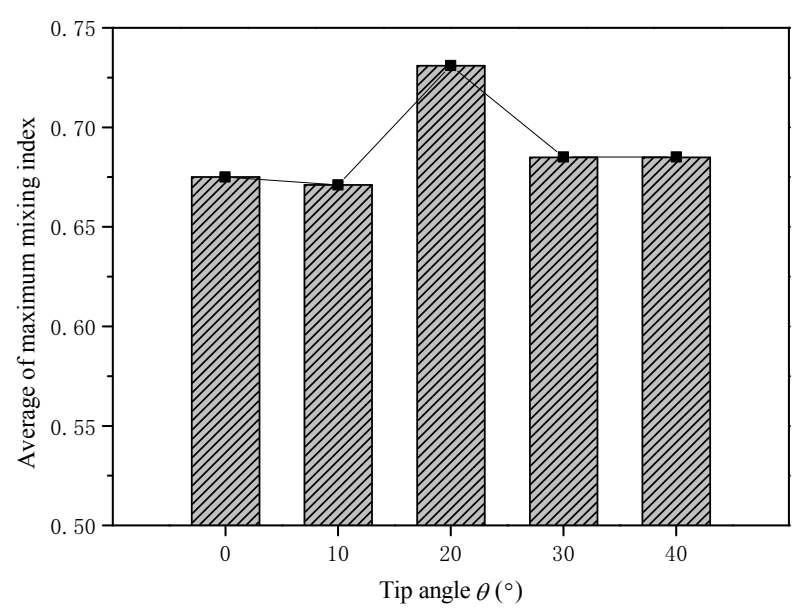

Fig.10 Average value of maximum mixing index in different tip angles

\section{Experiment \\ 4.1 Experiment Equipment}

In order to evaluate the mixing performance of screw rotors for the differential twin-screw kneader, a pair of screw rotors is machined when tip angle of male rotor is $0^{\circ}$ according to above analysis. The machined screw rotors are shown in Fig.11(a). Then one experimental apparatus of the twin-screw kneader is built up using the screw rotors. The experimental apparatus of the twin-screw kneader is mainly consisted of a motor, gearbox, screw rotors, screw barrel, screw barrel as well as a heating and temperature controlling system, etc.. As shown in Fig.12(b), the assembly relationship among these devices are as follows: frequency converter controls the motor, and the motor and gearbox are connected through the coupling, the two output shaft of the gear box are respectively connected with female and male screw rotors of the twin-screw kneader by coupling, so as to drive the twin-screw kneader. The material will be melt, sheared, mixed, then conveyed and extruded from the discharge port after the material are put inside the machine barrel through the feeding port. 


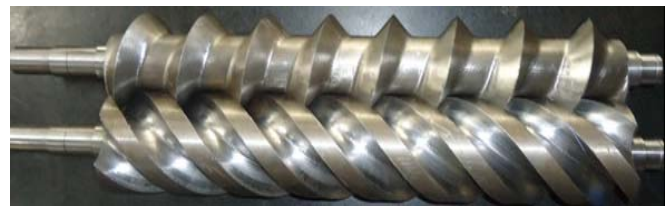

(a)

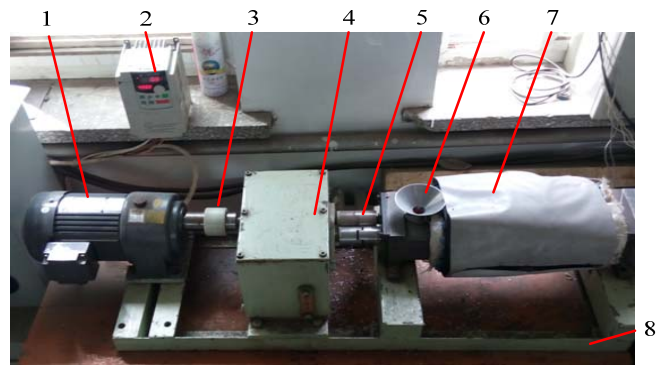

(b)

Fig.11 The novel twin-screw kneader system: (a) Machined screw rotors, (b) Twin-screw kneader system. 1-motor, 2-PI8000/8100 frequency converter, 3-coupling, 4-gearbox, 5-coupling, 6-screw rotors and barrel, 7-heating and temperature controlling system, 8 - bedplate

\subsection{Experiment Approach}

Color masterbatch is generally composed of colorants, carrier, dispersant composition. Masterbatch carrier are made of polypropylene, the density range of the PP is from 0.910 to $0.925 \mathrm{~g} / \mathrm{cm}^{3}$, the melting point is from $164^{\circ} \mathrm{C}$ to $170^{\circ} \mathrm{C}$, the temperature of thermal decomposition is at $300^{\circ} \mathrm{C}$ to $380^{\circ} \mathrm{C}$. Three kinds of raw materials with different color masterbatches are selected in the mixing performance experiment. The higher content of pigments or additives, and thermoplastic resin, a colorant is formed in the dispersion effect under plastic, resin raw material which has good dispersion and can be compatibility with coloring material of the additive. The colorant of red masterbatch is tonyred. The colorant of white masterbatch is calcium carbonate and the black masterbatch is using carbon black colorant. These three kind of the color masterbatches are shown in Fig. 12.

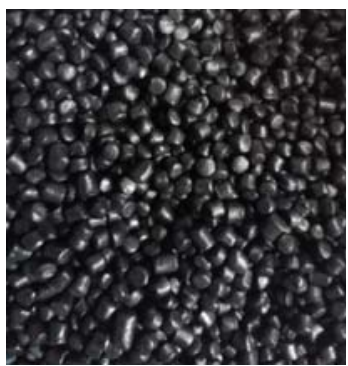

(a)

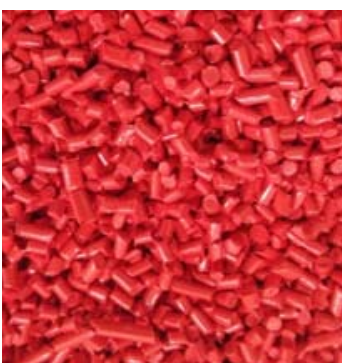

(b)

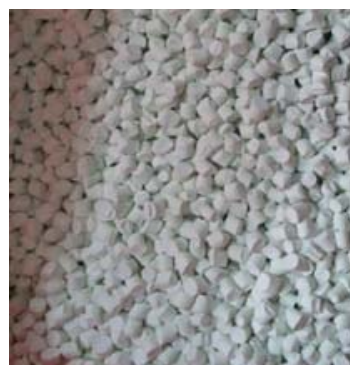

(c)

Fig. 12 Raw masterbatch material: (a) black masterbatch, (b) red masterbatch, (c) white masterbatch.

In order to compare the microscopic features of color masterbatch after mixing, it is necessary to observer the microscopic particles distribution of the raw masterbatch material before mixing. The optical microscope VHX-600E shown in Fig.13 (made by Keyence company in Japan) is used to observe the microscopic characteristics of topography for the observation of the experimental and extrusion materials sections.

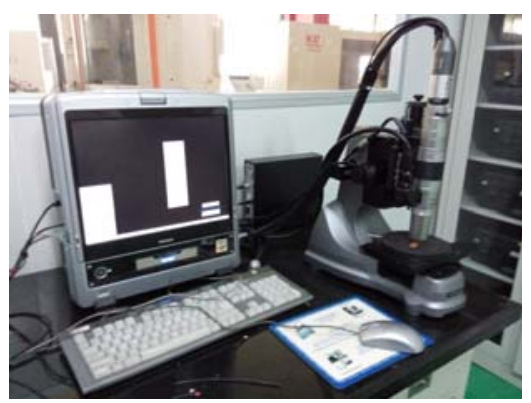

Fig.13 Optical microscope VHX-600E 
The surface morphology of the above three kinds of masterbatch materials are observed using the optical microscope VHX-600E after slicing, as shown in Fig.14.

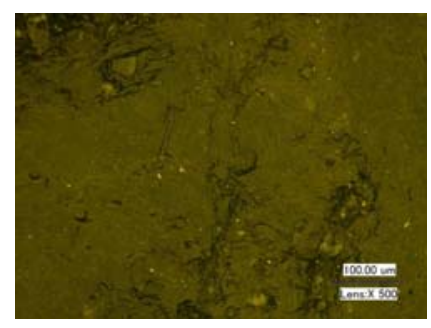

(a)

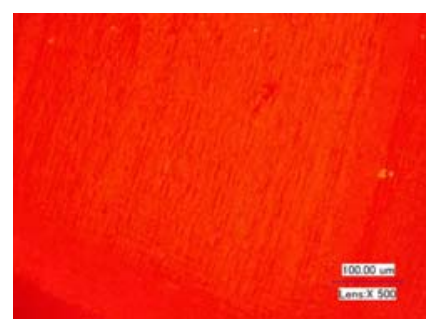

(b)

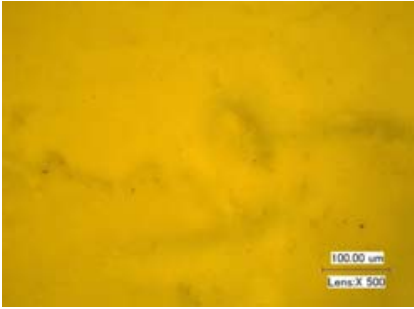

(c)

Fig.14 Micro-morphology of masterbatch material: (a) black masterbatch, (b) red masterbatch, (c) white masterbatch.

The purpose of the experiment is to evaluate the mixing performance of above screw rotors, and three kinds of different color masterbatch material were divided into three groups. The micro mixing results will be detected then to evaluate the mixing effect using the optical microscope. The rotation speed of the screw rotors is determined by the motor frequency. The reduction ratio of gearbox is $15: 1$. The rotation speed ratio of female and male rotor is $1: 4$. The rotation speed of the male rotor is $100 \mathrm{rpm}$ and that of female rotor is $25 \mathrm{rpm}$. Actually, it needs to premix before polymer material processing in the extrusion kneading machine. As the processing of raw materials, two kinds of different color masterbatch material should be mixed evenly. The premixing of red and white, red and black as well as black and white color masterbatch material are shown in Fig. 15(a),Fig.15(b) and Fig.15(c), respectively.

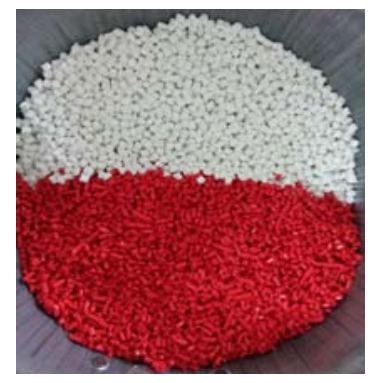

(a)

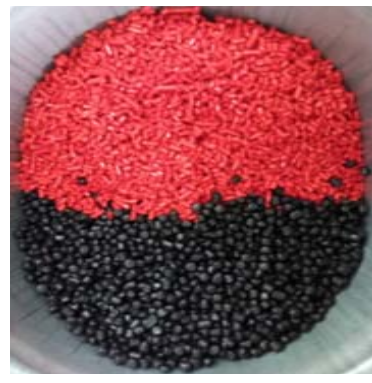

(b)

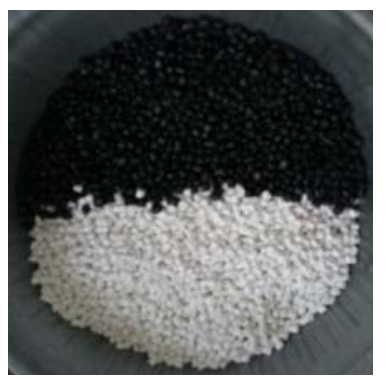

(c)

Fig.15 Premixing of masterbatch material: (a) Red and white, (b) Red and black, (c) Black and white.

\subsection{Results and analysis}

The die of experimental prototype have small circular holes, and the extruded molten material is a cylindrical granulated product of small diameter after cooling。 The extrusion products of different color masterbatch materials are shown in Fig. 16 in case of the temperature of screw barrel is $180^{\circ} \mathrm{C}$. It can be seen that the novel differential twin-screw kneader can successfully extrude molten material when reaching the melting temperature of color masterbatch.

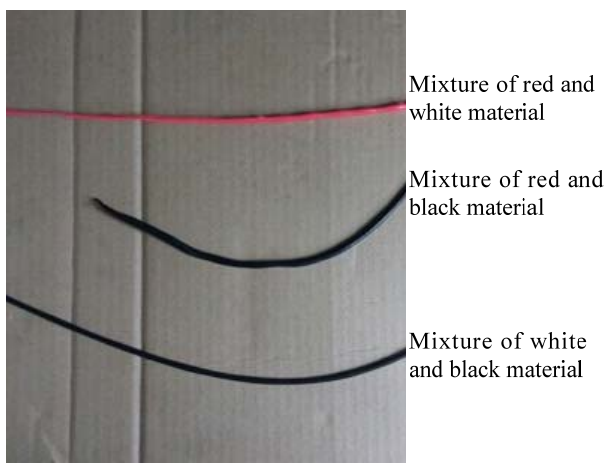

Fig.16 Extrusion products 
In order to verify the mixing performance of differential twin-screw kneader, the extrusion products in Fig. 16 were cut into slices to analyze the mixture using the optical microscope VHX-600E. Fig.17 shows the micro mixing of the extrusion products using the optical microscope after 500 times magnification.

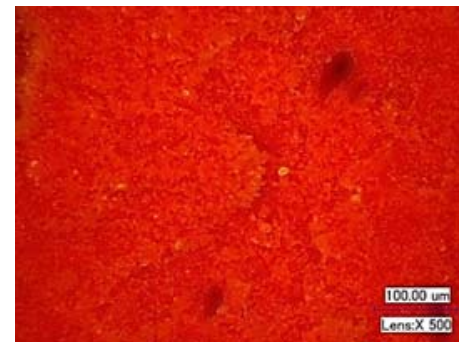

(a)

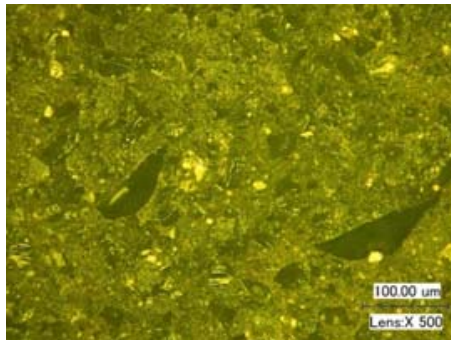

(b)

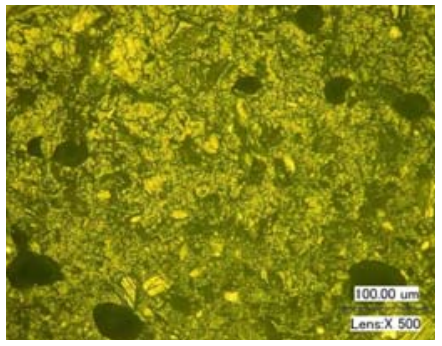

(c)

Fig.17 Mixed results of different masterbatch: (a) Red and white, (b) Red and black, (c) Black and white.

Figure 17(a) shows the mixing effect of red and white masterbatch. It can be seen that white calcium carbonate particles are more homogeneously mixed among red material under the high-powered microscope. Figure 17(b) is the mixing effect of red and black masterbatch. The largely black area is air bubble resulted by the incompletely discharge gas during the extrusion process in Fig.17 (b). It can be seen that carbon black particles and white particles of calcium carbonate are interphase mixed and more uniform as a whole from Fig.17(c). According to above mixing experiment results, the novel twin-screw kneader using differential twin-screw rotors as core working parts can be used as the mixed polymer processing.

\section{Conclusions}

In this paper, the mixing performance of the screw rotors for one novel differential intermeshing counter-rotating twin-screw kneader with different tip angles was analyzed using FEM along with MST implemented by POLYFLOW. Particle tracking technique is used to obtain the parameters along the particle trajectories, such as the residence time, segregation scale, shear rate, stretching rate, and the mixing index, then statistical analysis was carried out for flow field to estimate the distributive and dispersive mixing performance of the screw rotors. One experimental apparatus of the twin-screw kneader is built up using the screw rotors when tip angle of male rotor is $20^{\circ}$. Three kinds of different color masterbatch material are used to observe the mixing performance. Some conclusions can be drawn as followings:

1. The spatial flow pattern and trajectories of the particles indicate that particles not only exist the axial movement in the mixing process, but also cross flow between the upper and lower part of the flow channel, therefore, the particles continuously exchange between the two sides of the flow channel, which can improve the distributive mixing capability, and for the screw rotors, of which the rotational speed of male screw is four times higher than that of the female rotor, the male screw with a high rotating speed plays an important role in the mixing process.

2. In the process of statistical analysis, distributive mixing performance is estimated using the RTD and the segregation scale, while the dispersive mixing performance is evaluated using the average of the maximum shear rate and stretching rate as well as the mixing index. The results show that, when the tip angle is smaller than $20^{\circ}$, the distributive mixing performance of the screw rotors is better than that while the tip angle is greater than $20^{\circ}$, and the best distributive mixing performance can be achieved when the tip angle is $0^{\circ}$; for the dispersive mixing, although when the tip angle is less than $20^{\circ}$, the particles have experienced higher shear and stretching effect in the mixing process, the proportion of shear flow the particles experienced is higher than that while the tip angle is greater than $20^{\circ}$, and the optimal dispersive mixing performance is obtained when the tip angle is $20^{\circ}$. The simulation results can then be used to improve mixer design and optimize mixing stage.

3. According to the slice analysis results of three kinds of extrusion products under the high-powered microscope, it can be seen that color masterbatch have been homogeneously mixed in the twin-screw kneader, so the novel twin-screw kneader using differential twin-screw rotors as core working parts can be used as the mixed processing of different masterbatch. 


\section{Acknowledgements}

This work is supported by National Natural Science Foundation of China (No.51275553). The authors would like to take this opportunity to express their sincere appreciation.

\section{References}

Avalosse, T., Rubin, Y. and Fondin, L., Non-isothermal modeling of co-rotating and contra-rotating twin screw extruders, Journal of Reinforced Plastics and Composites, Vol.21, No.5(2002), pp.419-429.

Alsteens, B., Legat, V. and Avalosse, T., Parametric study of the mixing efficiency in a kneading block section of a twin-screw extruder, International Polymer Processing, Vol.19, No.3(2004), pp.207-217.

Böhme, G. and Wünsch, O., Analysis of shear-thinning fluid flow in intermeshing twin-screw extruders, Archive of Applied Mechanics,Vol.67,No.3 (1997), pp.167-178.

Brouwer, T., Todd, D.B. and Janssen, L.P.B.M., Flow characteristics of screws and special mixing enhancers in a co-rotating twin screw extruder. International Polymer Processing, Vol.17, No.1 (2002), pp.26-32.

Cheng, J.J. and Manas-Zloczower, I., Flow field characterization in a banbury mixer, International Polymer Processing, Vol.5, No.3 (1990), pp.178-183.

Cheng, H. and Manas-Zloczower, I., Distributive mixing in conveying elements of a ZSK-53 co-rotating twin screw extruder. Polymer Engineering and Science, Vol.38, No.6 (1998), pp.926-935.

Connelly, R.K. and Kokini, J.L., Examination of the mixing ability of single and twin screw mixers using 2D finite element method simulation with particle tracking, Journal of Food Engineering, Vol.79, No.3(2007), pp.956-969.

Djuric, D. and Kleinebudde, P., Impact of screw elements on continuous granulation with a twin-screw extruder. Journal of Pharmaceutical, Vol.97, No.11 (2008), pp.4934-4942.

Huang, H.X., Jiang, G. and Mao, S.Q., Microstructure and on-line shear viscosity of PP/nano-CaCO3 composites prepared by twin-screw extruder, Journal of Materials Science, Vol.41,No.15 (2006), pp.4985-4988.

Ishikawa, T., Amano, T., Kihara, S.I. and Funatsu, K., Flow patterns and mixing mechanisms in the screw mixing element of a co-rotating twin-screw extruder. Polymer Engineering and Science, Vol.42, No.5 (2002), pp.925-939.

Ishikawa, T., Kihara, S.I. and Funatsu, K., 3-D numerical simulations of nonisothermal flow in co-rotating twin screw extruders, Polymer Engineering and Science,Vol.40,No.2 (2000), pp.357-364.

Ishikawa, T., Nagano, F., Kajiwara, T. and Funatsu, K., Tip-clearance effect on mixing performance of twin screw extruders, International Polymer Processing, Vol.21, No.4(2006), pp.354-360.

Kajiwara, T., Nagashima, Y., Nakano, Y. and Funatsu, K., Numerical study of twin-screw extruders by three-dimensional flow analysis-development of analysis technique and evaluation of mixing performance for full flight screws, Polymer Engineering and Science, Vol.36, No.16(1996), pp.2142-2152.

Kumar, A., Ganjyal, G.M., Jones, D.D. and Hanna, M.A., Modeling residence time distribution in a twin-screw extruder as a series of ideal steady-state flow reactors, Journal of Food Engineering, Vol.84, No.3(2008), pp.441-448.

Potente, H., Kretschmer, K., Hofmann, J., Senge, M., Mours, M., Scheel, G. and Winkelmann, T., Process behavior of special mixing elements in twin-screw extruders. International Polymer Processing, Vol.16, No.4 (2001), pp.341-350.

Rathod, M.L., Kokini, J.L., Effect of mixer geometry and operating conditions on mixing efficiency of a non-Newtonian fluid in a twin screw mixer, Journal of Food Engineering, Vol.118,No.3 (2013),pp.256-265.

Shon, K., Bumm, S.H. and White, J.L., A comparative study of dispersing a polyamide 6 into a polypropylene melt in a Buss Kneader, continuous mixer, and modular intermeshing corotating and counter-rotating twin screw extruders, Polymer Engineering and Science,Vol.48, No.4 (2008),pp.755-766.

Wei, J., Li, G. and Zhang, G.H., Profiles Design, Optimization and geometric analysis of rotors of a novel continuous kneader reactor. Journal of Reinforced Plastics and Composites, Vol.29, No.15 (2010), pp.2279-2295.

Wei, J., Sun, Q., Sun, X. and Sun, W., A study on rotor profiles design for a novel twin-screw kneader. International Journal of Precision Engineering and Manufacturing, Vol.14, No.3 (2013), pp.451-459.

Wei, J., Zhang, G.H., Zhang, Q., Kim, J. and Lyu, S., Static load analysis of twin-screw kneaders. International Journal of Precision Engineering and Manufacturing, Vol.9, No.3 (2008), pp.59-63.

Wei J., Liang X.L., Chen D.B., Yang Y.L. and Zhou D.M., Evaluation of the mixing performance for one novel twin 
Wei, Chen and Zhou, Journal of Advanced Mechanical Design, Systems, and Manufacturing, Vol.9, No.3 (2015)

screw kneader with particle tracking, Polymer Engineering and Science, No.54.No.10(2014), pp.2407-2419. 